\title{
Marcação de tempo por surdos sinalizadores brasileiros***
}

\author{
Tense marking by Brazilian deaf signers
}

\author{
Aline Nascimento Crato* \\ Maria Silvia Cárnio**
}

*Fonoaudióloga. Mestranda em Ciências da Reabilitação Comunicação Humana do Departamento de Fisioterapia, Fonoaudiologia Terapia Ocupacional da Faculdade de Medicina da Universidade de São Paulo (FMUSP). Colaboradora do Laboratório de Investigação Fonoaudiológica em Leitura e Escrita. Endereço para correspondência: Av. Charles Schnneider, 1001 - Apto. 53C - São Paulo - SP - CEP 12040-000 (aline.crato@bol.com.br).

**Fonoaudióloga. Doutora em Semiótica e Linguística Geral pela Faculdade de Filosofia, Letras e Ciências Humanas (FFLCH - USP). Docente do Curso de Fonoaudiologia do Departamento de Fisioterapia, Fonoaudiologia e Terapia Ocupacional da FMUSP.

**Trabalho Realizado no Departamento de Fisioterapia, Fonoaudiologia e Terapia Ocupacional da FMUSP.

Artigo Original de Pesquisa

Artigo Submetido a Avaliação por Pares

Conflito de Interesse: não

Recebido em 21.06.2010. Revisado em 26.08.2010. Aceito para Publicação em 01.09.2010.

\begin{abstract}
Background: tense marking by deaf signers, in the Brazilian Sign Language and in written Portuguese. Aim: to analyze verbal tense inflection in written Portuguese, to verify the relationship between the performance in using verbal tense inflexion and the educational status, and to verify tense marking in the production of sentences in the Brazilian sign language and in written Portuguese. Method: participants were 18 deaf signers ranging in age from 15 to 23 years, and with an educational status varying from 3 rd to 7th grade of elementary school. Participants were assessed for their knowledge of nine action verbs. Following that, they were asked to elaborate three sentences with each verb using written Portuguese and using Brazilian sign language, one in the past tense, one in the present tense and one in the future tense. Data were analyzed qualitative and quantitatively. Results: Regarding the written productions, there was a predominance of the verb in the infinitive nominal form. Participants adequately used tense markers in most of the sentences produced when using the Brazilian sign language. Four subjects used sign language markers to indicate tense in their written sentences. There was a significant statistical relationship between the use of verbal inflexion in the present tense and higher educational status. Conclusion: the deaf participants of the study presented and adequate use of tense markers in most of the sentences produced using Brazilian sign language, however difficulty was observed when using written Portuguese. Key Words: Sign Language; Deafness; Evaluation; Language.
\end{abstract}

\section{Resumo}

Tema: marcação de tempo realizada por surdos sinalizadores na Língua Brasileira de Sinais e na Língua Portuguesa Escrita. Objetivos: analisar a flexão verbal de tempo na Língua Portuguesa Escrita, averiguar se existe relação entre o desempenho no uso da flexão verbal de tempo e grau de escolaridade e verificar os marcadores de tempo utilizados na produção de frases na Língua Brasileira de Sinais e na Língua Portuguesa Escrita. Métodos: participaram do estudo 18 surdos sinalizadores, com idade entre 15 e 23 anos e escolaridade de $3^{\mathrm{a}}$ a $6^{\mathrm{a}}$ série do Ensino Fundamental. Os indivíduos foram avaliados quanto ao conhecimento em Língua Brasileira de Sinais de nove verbos de ação, e em seguida foram orientados a elaborar três frases na Língua Portuguesa Escrita e na Língua Brasileira de Sinais com cada verbo, sendo uma no tempo passado, uma no presente e uma no futuro. Os dados foram avaliados qualitativa e quantitativamente. Resultados: nas frases escritas houve o predomínio do verbo na forma nominal do infinitivo. Os sujeitos utilizaram adequadamente os marcadores de tempo na maioria das frases expressas na Língua Brasileira de Sinais. Quatro sujeitos fizeram uso de marcadores utilizados na língua de sinais para indicar o tempo nas frases escritas. Houve relação estatisticamente significante entre o uso da flexão verbal do tempo presente com o aumento da escolaridade. Conclusão: os surdos do estudo utilizam adequadamente marcadores de tempo na maioria das frases expressas na Língua Brasileira de Sinais, mas apresentam dificuldade na Língua Portuguesa Escrita.

Palavras-Chave: Linguagem de Sinais; Surdez; Avaliação; Linguagem. 


\section{Introdução}

Pesquisas mencionam a influência da língua de sinais na produção escrita de surdos ${ }^{1-4}$. De acordo com a literatura a maioria dos erros cometidos no aprendizado de uma segunda língua se deve ao fato dos sujeitos se basearem na estrutura da primeira língua ${ }^{5-9}$ não sendo uma particularidade do surdo. Se as crianças surdas tivessem acesso à língua de sinais desde os primeiros anos de vida, o aprendizado da língua escrita, como segunda língua, seria adquirido de forma mais rápida e significativa, 10-12 uma vez que estudos internacionais ${ }^{13-16}$ enfatizam que existe relação entre a competência linguística em língua de sinais e a aquisição da escrita em crianças surdas.

No Brasil, ainda são restritos os estudos que relacionam o desempenho linguístico de surdos sinalizadores no uso da língua brasileira de sinais (Libras) e na língua portuguesa escrita. Pesquisas nacionais e internacionais sobre a produção escrita de surdos apontam a flexão verbal como um aspecto muito difícil para os mesmos ${ }^{3-4,14,17-}$ 19. Embora os verbos possam se apresentar lexicalmente corretos na escrita dos sujeitos, não há domínio das flexões de tempo no processo de construção frasal ${ }^{4,18}$. Na Libras, assim como em outras línguas de sinais, a marcação de tempo é expresso por meio de morfemas puros ${ }^{20-22}$, ou seja, não possui flexão verbal de tempo como ocorre na língua portuguesa.

Com base no exposto, o presente estudo teve por objetivos:

- analisar a flexão verbal de tempo na língua portuguesa escrita por surdos sinalizadores;

- averiguar se existe relação entre o desempenho dos surdos sinalizadores na flexão verbal de tempo da língua portuguesa escrita e grau de escolaridade; - verificar os marcadores de tempo utilizados na produção das frases na Libras e na língua portuguesa escrita.

Hipotetiza-se que a falta de conhecimento sobre as regras da língua portuguesa faz com que os surdos tenham dificuldade no uso da flexão verbal de tempo e utilizem marcadores da Libras.

\section{Método}

Esta pesquisa, avaliativa prospectiva, foi aprovada pela Comissão de Ética para Análise de Projetos de Pesquisa (CAPPesq) da Diretoria Clínica do Hospital das Clínicas e da Faculdade de Medicina da Universidade de São Paulo, sob protocolo número 0708/07. O Termo de Consentimento Livre e Esclarecido foi assinado pelos participantes ou seus responsáveis (no caso de adolescentes).

Os participantes foram selecionados de uma instituição pública especializada no atendimento de surdos do interior de São Paulo. De um total de 98 surdos, somente $18(18,4 \%)$ preencheram os critérios de inclusão deste estudo. A faixa etária dos sujeitos variou de 15 a 23 anos (média de 18:2 anos), dos quais $12(66,66 \%)$ eram do gênero masculino e seis $(33,33 \%)$ do feminino. Todos apresentavam perda auditiva neurossensorial de grau profundo bilateral (média de 500, 1000 e 2000 Hz maior que 91 dBNA) congênita ou adquirida antes de um ano de idade e utilizavam a língua de sinais, há mais de quatro anos, como meio de comunicação. Os participantes frequentavam classes comuns e tinham escolaridade entre $3^{\mathrm{a}}$ e $6^{\mathrm{a}}$ série do Ensino Fundamental. Todos eram filhos de pais ouvintes e não apresentavam comprometimentos associados à surdez.

Os verbos usados na avaliação dos sujeitos foram retirados do Caderno Ilustrado de Verbos ${ }^{23}$. Para a seleção dos verbos, procurou-se evitar aqueles nos quais o sinal correspondente na Libras fosse igual ou semelhante ao substantivo, como por exemplo, o sinal de "DIRIGIR" e "CARRO". Além disso, foram selecionados verbos que se enquadrassem na classificação de Quadros ${ }^{20} \mathrm{em}$ verbos simples, ou seja, que não incorporam afixos locativos e necessitam do movimento de apontar para indicar o sujeito. Em relação ao Português, foram escolhidos verbos regulares e dissílabos, segundo os critérios da Gramática da Língua Portuguesa $^{24}$. Deste modo, foram selecionados os seguintes verbos para a avaliação: beber, comer, correr, fechar, jogar, limpar, nadar, pintar e pular.

Inicialmente os sujeitos responderam a um questionário informativo referente à trajetória linguística e escolar dos mesmos.

Em seguida foram apresentadas aos participantes as ilustrações das cartelas de verbos selecionados e solicitada a realização do sinal correspondente a cada verbo, na Libras, para que fosse garantido o real entendimento dos mesmos. 
Posteriormente, os sujeitos foram instruídos a escrever o nome do verbo e elaborar três frases escritas e na Libras, sendo respectivamente uma no tempo passado, uma no presente e uma no futuro.

As avaliações foram filmadas e realizadas individualmente pela pesquisadora, a qual transmitiu todas as instruções por meio da Libras. As gravações foram transcritas e os registros categorizados para posterior análise qualitativa e quantitativa.

Critérios para a análise dos resultados

Para a análise da classificação dos tempos verbais e dos marcadores de tempo utilizados nas produções escritas usou-se a Gramática da Língua Portuguesa ${ }^{24}$.

Os marcadores de tempo utilizados nas produções das frases em Libras foram categorizados em: contexto, advérbio de tempo e adjunto adverbial de tempo. As frases do presente que não utilizaram marcadores de tempo foram consideradas adequadas, pois segundo Felipe ${ }^{21}$ na Libras a referência ao presente não possui indicação temporal.

Um instrutor surdo da FENEIS foi convidado a analisar as filmagens das avaliações das frases produzidas em Libras de cinco sujeitos sorteados aleatoriamente, com o objetivo de medir o índice de concordância da avaliação das frases em Libras realizada pela pesquisadora e pelo instrutor de Libras, utilizando-se para tanto o Índice de Concordância de Kappa.

Para comparar o desempenho dos sujeitos no uso das flexões verbais de tempo nas frases da Língua Portuguesa Escrita e sua relação com o grau de escolaridade foram utilizados os testes de Wilcoxon e de Mann-Whitney.

Por questão de quantidade amostral foi realizado um agrupamento das $3^{\mathrm{a}} \mathrm{s}$ e $4^{\mathrm{a}} \mathrm{s}$ e das $5^{\mathrm{a}} \mathrm{s}$ e $6^{\text {a }}$ s séries, a fim de se obter uma melhor análise.
Foi adotado um nível de significância de 0,05 (5\%), e todos os intervalos de confiança construídos ao longo do trabalho foram feitos com 95\% de confiança estatística.

\section{Resultados}

O índice de concordância Kappa entre a avaliação da pesquisadora e do instrutor de Libras em relação ao tempo das frases e dos marcadores utilizados para indicar o tempo em Libras foram estatisticamente significantes e considerados ótimos (acima de $81 \%$ ).

Dessa forma, os resultados apresentados abaixo foram analisados somente pela pesquisadora.

No Quadro 1 estão expostos os resultados das flexões verbais de tempo utilizadas pelos surdos na elaboração de frases escritas no passado, presente e futuro.

Nas frases escritas no tempo passado com os verbos flexionados corretamente foi utilizado o pretérito perfeito do modo indicativo acompanhado do adjunto adverbial "passado". Os verbos flexionados inadequadamente neste tempo foram escritos no presente do modo indicativo.

Todos os sujeitos que produziram as frases no tempo presente flexionaram os verbos na segunda pessoa do singular do modo indicativo, apesar de utilizarem o pronome "eu" na maioria das frases. Os verbos flexionados inadequadamente neste tempo foram escritos no pretérito perfeito do modo indicativo.

Nas frases do tempo futuro nenhum dos sujeitos realizou a flexão verbal de tempo corretamente. Os verbos flexionados inadequadamente neste tempo foram escritos no presente $(15,5 \%)$ e no pretérito perfeito $(1,2 \%)$ do modo indicativo.

QUADRO 1. Distribuição da flexão verbal de tempo utilizada pelos sujeitos nas frases escritas nos tempos passado, presente e futuro

\begin{tabular}{|c|c|c|c|c|c|c|c|c|}
\hline \multirow[t]{2}{*}{ Tempos } & \multicolumn{2}{|c|}{$\begin{array}{l}\text { Verbo Flexionado } \\
\text { Adequadamente }\end{array}$} & \multicolumn{2}{|c|}{$\begin{array}{c}\text { Verbo } \\
\text { Flexionado } \\
\text { Inadequadamente }\end{array}$} & \multicolumn{2}{|c|}{$\begin{array}{c}\text { Verbo na Forma } \\
\text { Nominal do } \\
\text { Infinitivo }\end{array}$} & \multicolumn{2}{|c|}{ Não Elaborou a Frase } \\
\hline & $\mathrm{N}$ & $\%$ & $\mathrm{~N}$ & $\%$ & $\mathrm{~N}$ & $\%$ & $\mathrm{~N}$ & $\%$ \\
\hline passado & 2 & $1,2 \%$ & 33 & $20,4 \%$ & 63 & $38,9 \%$ & 64 & $39,5 \%$ \\
\hline presente & 27 & $16,7 \%$ & 2 & $1,2 \%$ & 47 & $29 \%$ & 86 & $53,1 \%$ \\
\hline futuro & 0 & $0 \%$ & 27 & $16,7 \%$ & 41 & $25,3 \%$ & 94 & $58 \%$ \\
\hline TOTAL $(\mathrm{N}=486)$ & 29 & $6 \%$ & 62 & $12,8 \%$ & 151 & $31 \%$ & 244 & $50,2 \%$ \\
\hline
\end{tabular}

Legenda: N: Número de frases 
O teste de Wilcoxon foi aplicado para comparar o uso das flexões verbais de tempo nas produções das frases escritas no passado, presente e futuro (Tabela 1).

Nota-se melhor desempenho na flexão verbal do tempo presente em relação ao passado e ao futuro, sendo observada diferença estatisticamente significante.

Para comparar os sujeitos de diferentes graus de escolaridade em relação a seus desempenhos no uso das flexões verbais de tempo foi utilizado o teste de Mann-Whitney (Tabela 2).

As flexões verbais do tempo futuro não foram incluídas nesta análise, pois nenhum dos sujeitos flexionou adequadamente os verbos na elaboração das frases deste tempo.

Observa-se que existiu diferença estatisticamente significante entre o grau de escolaridade e a flexão verbal do tempo presente, onde nota-se que os sujeitos de $5^{\mathrm{a}}$ e $6^{\mathrm{a}}$ séries utilizam mais frequentemente esta flexão.

Quatro sujeitos da pesquisa utilizaram marcadores de tempo na produção das frases escritas para suprir a ausência ou dificuldade no emprego da flexão verbal de tempo. Estes sujeitos utilizaram o advérbio de tempo "antes" e o adjunto adverbial "passado" para marcar o tempo das frases do passado. Nas frases do presente os sujeitos utilizaram o advérbio de tempo "hoje" e nas frases do futuro empregaram o advérbio de tempo "depois" e o adjunto adverbial "futuro".

Nas frases expressas em Libras no tempo passado houve $74,7 \%$ de produções adequadas, sendo que o marcador de tempo empregado com maior frequência foi o adjunto adverbial (33,3\%), representado pelo sinal de "PASSADO"; seguido do contexto $(24,7 \%)$, representado pelos sinais de "EU CRIANÇA" ou pelo encadeamento do discurso referente ao passado (Exemplo: "EU GORD@*CORRER EMAGRECER MAGR@*BO@*")e de advérbios de tempo (16,7\%), representado pelos sinais de "ANTIGAMENTE", "JÁ", "ONTEM" e "ANTES". Nas frases inadequadamente no passado $23,5 \%$ foram produzidas sem marcadores de tempo e $1,2 \%$ foram expressas no tempo futuro, sendo que $0,6 \%$ não foram elaboradas.

No tempo presente os sujeitos produziram $90,1 \%$ de frases adequadas na Libras. O marcador de tempo utilizado foi o advérbio de tempo (48,8\%), representado pelos sinais de "AGORA" e "HOJE". As demais frases do presente $(41,3 \%)$ consideradas adequadas foram elaboradas com ausência de marcador. Houve $6,8 \%$ de produções frasais inadequadas no presente, sendo que $6,2 \%$ foram expressas no tempo futuro e $0,6 \%$ no passado, sendo que $3,1 \%$ não foram realizadas.
TABELA 1. Comparação do uso das flexões verbais de tempo utilizadas pelos sujeitos nas produções das frases escritas nos tempos passado, presente e futuro.

\begin{tabular}{c|c|c}
\hline Flexão Verbal & Passado & Presente \\
\hline presente & $0,027^{*}$ \\
futuro & 0,317 & $0,018^{*}$ \\
\hline
\end{tabular}

Legenda: *: p-valores considerados estatisticamente significantes; Nível de significância: $\mathrm{p} \leq 0.05$.

TABELA 2. Comparação entre os sujeitos de diferentes graus de escolaridade e seus desempenhos no uso das flexões verbais dos tempos passado e presente.

\begin{tabular}{c|ccc|c|c}
\hline \multirow{2}{*}{$\begin{array}{c}\text { Série versus Flexão } \\
\text { verbal de Tempo }\end{array}$} & \multicolumn{2}{|c|}{ Passado } & \multicolumn{2}{c}{ Presente } \\
\cline { 2 - 5 } & $3^{\mathrm{a}} \mathrm{e} 4^{\mathrm{a}}$ & $5^{\mathrm{a}} \mathrm{e} 6^{\mathrm{a}}$ & $3^{\mathrm{a}} \mathrm{e} 4^{\mathrm{a}}$ & $5^{\mathrm{a}} \mathrm{e} 6^{\mathrm{a}}$ \\
\hline média & 0,00 & 0,22 & 0,22 & 2,78 \\
mediana & 0 & 0 & 0 & 3 \\
desvio padrão & 0,00 & 0,67 & 0,67 & 2,59 \\
Q1 & 0 & 0 & 0 & 0 \\
Q3 & 0 & 0 & 0 & 4 \\
N & 9 & 9 & 9 & 9 \\
IC & $-\mathrm{x}-$ & 0,44 & 0,44 & 1,69 \\
p-valor & \multicolumn{2}{c}{0,317} & \multicolumn{2}{c}{$0,012^{*}$} \\
\hline
\end{tabular}

Legenda: *: p-valores considerados estatisticamente significantes; IC: Intervalo de Confiança; N: Número de sujeitos; Nível de significância: $\mathrm{p} \leq 0.05$; Q1: $1^{\circ}$ quartil que mostra a distribuição até $25 \%$ da amostra; Q3: $3^{\circ}$ quartil que mostra a distribuição até $75 \%$ da amostra; -X-: Não foi possível utilizar cálculo estatístico.

Nas frases expressas em Libras no tempo futuro houve $71 \%$ de produções adequadas, sendo que o marcador de tempoutilizadocommaior frequênciafoi oadjuntoadverbial (39,5\%), representado pelo sinal de "FUTURO, seguido do contexto (18,5\%), representado pelos sinais de "EU VELH@*" ou pelo encadeamento do discurso referente ao futuro (Exemplo: "FILH@* VIDEOGAME JOGAR DAR") e de advérbios de tempo (13\%), representados pelos sinais de "DEPOIS" e "AMANHÃ". Nesse tempo verbal, observou-se a presença de $21,6 \%$ de produções inadequadas, sendo 19,1\% devidoà ausência de marcador e 2,5\% expressas no tempo passado, sendo que 7,4\% não foram produzidas.

\section{Discussão}

A maioria dos verbos empregados nas frases produzidas na Língua Portuguesa foi escrita na forma nominal do infinitivo. Tais resultados corroboram a literatura $^{3-4}$ que mencionam o predomínio do verbo no infinitivo na produção escrita de surdos. 
Houve diferença estatisticamente significante no desempenho dos sujeitos quanto às frases escritas no uso de flexão verbal do tempo presente em relação ao passado e ao futuro. O melhor desempenho no uso do tempo presente pode ter ocorrido pelo fato do contexto da avaliação (elaboração de frases) privilegiar esse tempo, assim como a produção de narrativa favorece o uso do passado. Outra explicação para este fato é que as frases do presente dos sujeitos que não utilizaram marcadores em nenhum dos tempos foram consideradas corretas, pois de acordo com a literatura este tempo geralmente não possui especificação temporal $^{21}$.

Nenhum dos sujeitos realizou a flexão verbal nas frases do futuro. Pesquisadores da língua portuguesa vêm evidenciando o declínio no uso do tempo futuro sintético em detrimento da perífrase na língua portuguesa ${ }^{25}$. Dentre as frases produzidas no futuro não foi constatado o uso de perífrase.

Outro dado que chama a atenção é o número de frases não elaboradas na língua portuguesa escrita, demonstrando que o tempo de escolaridade dos sujeitos ( 9 a 18 anos) não foi suficiente para que se sentissem aptos a produzirem frases na segunda língua. Deve-se levar em consideração que todos os sujeitos iniciaram o processo escolar sem uma língua estabelecida. Segundo a literatura o atraso na aquisição de língua pode lentificar o processo de aquisição da escrita ${ }^{12,14-15,19}$.

\section{Referências Bibliográficas}

1. Mayer C. Shaping at the Point of Utterance: An Investigation of the Composing Processes of the Deaf Student Writer. J Deaf Stud. Deaf Educ. 1999;4(1):37-49.

2. Mayer C, Akamatsu CT. Deaf Children Creating Written Texts: Contributions of American Sign Language and Signed Forms of English. Am Ann Deaf. 2000;145(5):394-403.

3. Wolbers KA. Using balanced and interactive writing instruction to improve the higher order and lower order writing skills of deaf students. J Deaf Stud. Deaf Educ. 2008;13(2):257-77.
A relação estatisticamente significante entre o uso da flexão verbal do tempo presente com o aumento do grau de escolaridade corrobora a literatura que menciona que a flexão verbal de tempo torna-se adequada com o avanço da escolaridade e consequentemente ampliação do conhecimento da língua portuguesa ${ }^{4,18,26}$.

Os marcadores de tempo utilizados pelos surdos na elaboração das frases na Libras corroboram a literatura $^{20-22}$ que menciona o uso de advérbios de tempo ou de um item lexical para indicar o tempo, uma vez que esta língua não possui flexão verbal de tempo.

Os advérbios e adjuntos adverbiais de tempo utilizados como marcadores de tempo na elaboração das frases escritas demonstram que os participantes estão transpondo a estrutura da Libras para a língua portuguesa escrita. De acordo com a literatura é comum o uso de traços da primeira língua quando se está adquirindo uma segunda língua ${ }^{5-9,12}$.

O uso de marcadores utilizados na Libras para a escrita das frases do Português é um dado relevante, pois apesar dos sujeitos apresentarem dificuldade em realizar a indicação de tempo no verbo, têm conhecimento da importância de sua marcação para explicitar na frase o momento em que ocorreu a ação, demonstrando indícios de habilidades metalinguísticas.

\section{Conclusão}

Os surdos do estudo utilizam adequadamente os marcadores de tempo na maioria das frases elaboradas em Libras, mas apresentam dificuldade em marcar o tempo na língua portuguesa escrita. Os resultados indicam melhor desempenho dos sujeitos no uso da flexão verbal de tempo com o aumento do grau da escolaridade.

4. Crato AN, Cárnio MS. Análise da flexão verbal de tempo na escrita de surdos sinalizadores. Rev Bras Ed. Esp. $2009 ; 15(2): 233-50$.

5. Mayer C, Wells G. Can the linguistic interdependence theory support a bilingual-bicultural model of literacy education for deaf students? J Deaf Stud. Deaf Educ. 1996;1(2):93-107.

6. Wang W, Wen Q. L1 Use in the L2 Composing Pprocess: An Exploratory Study of 16 Chinese EFL Writers. J Second Language Writing. 2002;11(3):225-46. 
7. Woodall BR. Laguage-Switching: Using the First Language While Writing in a Second Language. J Second Language Writing. 2002;11(1):7-28.

8. Singleton JL, Morgan D, DiGello E, Wiles J, Rivers R.Vocabulary use by low, moderate, and high ASL-proficient writers compared to hearing ESL and monolingual speakers. J Deaf Stud. Deaf Educ.2004;9(1):86-103

9. Puente A, Alvarado JM, Herrera V. Fingerspelling and sign language as alternative codes for reading and writing words for Chilean deaf signers. . Am Ann Deaf. 2006;151 (3):299-310.

10. Ferreira S, Cárnio MS. Interferências do Acesso às Distintas Modalidades Linguísticas no Processo de Escrita Espontânea do Surdo Inserido no Contexto de Educação Especial. Pró-Fono. 1999;11(2):38-45.

11. Caporali AS, Lacerda CBF, Marques PL. Ensino de língua de sinais a familiares de surdos: enfocando a aprendizagem. Pró-Fono. 2005;17(1):89-98.

12. Hermans D, Knoors H, Ormel E, Verhoeven L. Modeling reading vocabulary learning in deaf children in bilingual education programs. J Deaf Stud. Deaf Educ. 2008, 13(2):155-74

13. Strong M, Prinz PM. A study of the relationship between American Sign Language and English literacy. J Deaf Stud. Deaf Educ. 1997;2(1):37-46.

14. Wilbur, RB. The use of ASL t support the development of English and literacy. J Deaf Stud. Deaf Educ. 2000;5(1):81104.

15. Koutsoubou M, Herman R, Woll B. Bilingual language profiles of deaf students: an analysis of the written narratives of three deaf writers with different language proficiencies. Deafness Educ. Int. 2006;8(3):144-68.
16. Rathmann C, Mann W, Morgan G. Narrative structure and narrative development in deaf children. Deafness and Educ Int. 2007;9(4):187-96.

17. Fabbretti D, Volterra V, Pontecorvo C. Written language abilities in deaf Italians. J Deaf Stud. Deaf Educ. 1998;3(3): 231-244.

18. Fernandes E. Linguagem e surdez. Porto Alegre: Artmed, 2003.

19. Jenny L. Singleton JL, Morgan D, DiGello E, Wiles J, Rivers R. Vocabulary use by low, moderate, and high ASLproficient writers compared to hearing ESL and monolingual speakers. J Deaf Stud. Deaf Educ. 2004;9(1):86-103.

20. Quadros RM, Karnopp LB. Língua de sinais brasileira: estudos linguísticos. Porto Alegre: Artmed; 2004.

21. Felipe TA. Libras em contexto: curso básico. 5.ed. Rio de Janeiro: Libregraf; 2005.

22. Burman D, Nunes T, Evans D. Writing profiles of deaf children taught through British Sign Language. Deafness Educ. Int. 2007;9(1):2-23.

23. Quintano-Neira, PRN, Cárnio MS. Caderno Ilustrado de Verbos: um recurso pedagógico para o desenvolvimento da Língua portuguesa. Barueri, SP: Pró-Fono; 2005.

24. Neto PC, Infante U. Gramática da Língua Portuguesa. São Paulo: Scipione; 2003.

25. Cunha CF, Cintra L. Breve Gramática do Português Contemporâneo. Lisboa: Ed João Sá da Costa; 1985.

26. Gonçalo SF. Perfil da produção escrita e da trajetória escolar de alunos surdos de Ensino Médio. [dissertação]. São Paulo (SP): Faculdade de Educação da Universidade de São Paulo; 2004. 